\title{
Special issue on pattern recognition and information processing using neural networks
}

\author{
F.-C. Sun $\cdot$ Y. Tan $\cdot$ Cong Wang
}

Published online: 14 June 2009

(C) Springer-Verlag 2009

\section{Preface}

Neural network techniques have proven to be flexible in pattern recognition and information processing in complex environments. They typically include BP networks, RBF networks, support vector machine (SVM) and other similar biologically motivated models. The neural network techniques are able to enhance recognition accuracy, and have found applications in real-world environments.

This special issue addresses neural network techniques in pattern recognition and information processing problems.

The first paper "Kernel based improved discriminant analysis and its application to face recognition," coauthored by Dake Zhou and Zhenmin Tang, presents a variant of KDA called kernel-based improved discriminant analysis (KIDA). In the proposed framework, original samples are projected firstly into a feature space by an implicit nonlinear mapping. After reconstructing betweenclass scatter matrix in the feature space by weighted

F.-C. Sun $(\bowtie)$

State Key Lab of Intelligent Technology and Systems,

Department of Computer Science and Technology,

Tsinghua University, 100084 Beijing, China

e-mail: fcsun@tsinghua.edu.cn

Y. Tan

Key Laboratory of Machine Perception (MOE),

Department of Machine Intelligence, School of Electronics,

Engineering and Computer Science, Peking University,

100871 Beijing, China

C. Wang

College of Automation Science and Engineering,

South China University of Technology,

510641 Guangzhou, China

e-mail: wangcong@scut.edu.cn schemes, the kernel method is used to obtain a modified Fisher criterion. Finally, simultaneous diagonalization technique is employed to find lower dimensional nonlinear features with significant discriminant power. The second paper "A Novel Application of a Self-Organizing Network for Recognition of Facial Expressions from Contours," coauthored by W.F. Gu, Y.V. Venkatesh, and C. Xiang, proposes a self-organizing network for recognizing facial expressions using biologically plausible features: contours of face and its components. Experimental results show that the recognition accuracy of the presented algorithm is superior to that of other algorithms in the literature on the Japanese Female Facial Expression (JAFFE) database. The third paper "An Automatic Fuzzy C-Means Algorithm for Image Segmentation," co-authored by Yanling Li and Yi Shen, proposes a novel fuzzy clustering algorithm for automatically grouping the pixels of an image into different homogeneous regions when the number of clusters is not known a priori. The new algorithm initiates the first two centroids of clusters by a method based on hard c-means algorithm and automatically determines the appropriate cluster number for image segmentation.

The fourth paper "Content Based Image Classification with Wavelet Relevance Vector Machines," co-authored by Arvind Tolambiya, S. Venkataraman, and Prem K. Kalra, introduces the use of relevance vector machines (RVM) for content-based image classification and compares it with the conventional SVM approach. The authors also propose a new wavelet-based feature extraction method that extracts less number of features as compared to other wavelet-based feature extraction methods. The fifth paper "A Three-layer Back-propagation Neural Network for Spam Detection Using Artificial Immune Concentration," co-authored by Guangchen Ruan and Ying Tan, uses a three-layer back-propagation neural network 
for spam detection. In the proposed concentration-based feature construction (CFC) approach, "self" concentration and "non-self" concentration are constructed to form a two-element concentration vector for expressing the e-mail efficiently. A three-layer neural network with two-element input is then employed to classify e-mails automatically. The sixth paper "k-Top Scoring Pair Algorithm for Feature Selection in SVM with Applications to Microarray Data Classification," co-authored by Sejong Yoon and Saejoon Kim, conducted a fair comparison between TSP and SVMRecursive Feature Elimination (SVM-RFE) as the feature selection method for SVM. The authors also compared kTSP with a couple of ensemble methods based on SVM. Results on ten public domain microarray data indicated that TSP family classifiers serve as good feature selection schemes which may be combined effectively with other classification methods.

The seventh paper "Optimization of Silicon Solar Cell Fabrication Based on Neural Network and Genetic Programming Modeling" is co-authored by Hyeon Bae, TaeRyong Jeon, Sungshin Kim, Hyun-Soo Kim, Dongseop Kim, Seung-Soo Han, and Gary S. May. Two modeling approaches, neural network and genetic programming, are employed to model the crucial relation for the consecutively connected two processes in solar cell fabrication for comparison purpose. The eighth paper "An Unbiased LSSVM Model for Classification and Regression," coauthored by Hongqiao Wang, Fuchun Sun, Yanning Cai, Linge Ding, and Ning Chen, aims at the common SVM's biased disadvantage and the computational complexity, and proposed an unbiased least square SVM model. Based on this model, a fast learning algorithm using Cholesky factorization is designed according to the characteristic of kernel function matrix. The experimental results of correct recognition rate and the fitting precision testify that the unbiased LSSVM model has good universal ability and fitting accuracy, better generalization capability and stability, and have a great improvement in learning speed.
The last paper "Relational Multiple Kernel Learning from Sets of Partially Matching Image Features," coauthored by Siyao Fu, Zengguang Hou, Zize Liang, Fengshui Jing, Min Tan and Qi Zuo, shows that multiple kernel learning problems can be solved efficiently by inducing logical rules. The authors use relational learning as an efficient method for solving correspondence measurement as well as automatically generating rules for acceptable shape variations from training data. Through iteratively constructing rules by a modified FOIL algorithm and performing the corresponding multiple kernel optimizations, the presented approach realizes an additive model on the feature space induced by the obtained rules. Experimental results show that the proposed method is accurate and significantly more efficient than current approaches.

The neural network-based intelligent computing technologies include a range of techniques such as artificial intelligence, perceptual and pattern recognition, evolutionary and adaptive computing, informatics processing theories and applications, computational neuroscience and bioscience, soft computing, case based and constrained reasoning, agents, networking and computer supported cooperative working, human computer interface issues, etc. This special session brings together researchers and practitioners in the area of theory, design, implementation, and applications of neural network-based pattern recognition and information processing. The successful applications of neural networks in pattern recognition and intelligent information processing suggest that this direction is likely to play an important role in science and engineering.

Finally, we hope this special issue will be a valuable resource for neural network-based pattern recognition and information processing.

Guest Editors

Fuchun Sun

Ying Tan

Cong Wang 